\title{
Crohn's disease at radiological imaging: focus on techniques and intestinal tract
}

\author{
Giuseppe Cicero, Silvio Mazziotti \\ Section of Radiological Sciences, Department of Biomedical Sciences and Morphological and Functional Imaging, University of Messina, \\ Messina, Italy
}

Over recent years, inflammatory bowel diseases have become an issue of increased attention in daily clinical practice, due to both a rising incidence and improved imaging capability in detection. In particular, the diagnosis of Crohn's disease is based on clinical picture, laboratory tests and colonoscopy with biopsy. However, colonoscopic evaluation is limited to the mucosal layer. Thus, imaging modalities play a pivotal role in enriching the clinical picture, delivering information on intestinal and extraintestinal involvement. All the imaging modalities can be employed in evaluation of Crohn's disease patients, each of them with specific strengths as well as limitations. In this wide selection, the choice of a proper diagnostic framework can be challenging for the clinician. Therefore, the aim of this work is to offer an overview of the different imaging techniques, with brief technical details and diagnostic potential related to each intestinal tract. (Intest Res 2021;19:365-378)

Key Words: Crohn disease; Fluoroscopy; Ultrasound; Computed tomography; Magnetic resonance imaging

\section{INTRODUCTION}

Inflammatory bowel diseases (IBDs) include 3 idiopathic and chronic intestinal conditions: Crohn's disease (CD), ulcerative colitis (UC) and indeterminate colitis. Their onset relies on a combination of genomic predisposition and environmental factors that lead to an altered immunoresponse to the intestinal microbiota. ${ }^{1-4}$ In particular, CD is characterized by transmural involvement of the bowel wall and it can affect any section of the gastrointestinal tract, with higher prevalence in the ileocolonic segments. Typical symptoms include fever, chronic diarrhea, fatigue and weight loss. However, concurrent involvement of different system and organs is common, with possible hepato-pancreatobiliary, renal, musculoskeletal, dermatologic, pulmonary and ocular manifestations. ${ }^{5,6}$ Ileocolo-

Received August 8, 2020. Revised October 2, 2020. Accepted October 2, 2020 Correspondence to Giuseppe Cicero, Department of Biomedical Sciences and Morphological and Functional Imaging, University of Messina, Via Consolare Valeria, 1, Messina 98100, Italy. Tel: +39-90-221-3092, Fax: +3990-221-3720,E-mail: gcicero@unime.it noscopy with biopsy is considered the reference standard for achieving a definitive diagnosis, as it can access hard-to-reach areas of the small intestine and can replace surgery in treatment of strictures through balloon dilation. ${ }^{7}$

However, the main limits include invasiveness, related risks (i.e., bowel perforation) and a partial evaluation, limited to the mucosal surface. ${ }^{8}$

Therefore, radiological imaging is of paramount importance in the estimation of the whole intestinal involvement, not only for the initial evaluation, but also for follow-up monitoring and treatment response assessment. ${ }^{9,10}$ Beyond the mere detection of intestinal lesions, it can provide useful data concerning characteristics of bowel wall thickening, which is helpful in distinguishing active inflammation from fibrosis, along with and valuable information on the surrounding tissues, where complications arise. ${ }^{11}$ The constant development of novel technical tools and techniques is a benefit for improving the patient's clinical framework. On the other hand, the different imaging modalities and the number of tools and techniques they are provided with can be confounding while establishing the prop- 
Table 1. Imaging Modalities and Crohn's Disease: Synoptic Table

\begin{tabular}{|c|c|c|c|c|}
\hline Imaging modality & Advantages & Disadvantages & Additional benefits & Intestinal applications \\
\hline Fluoroscopy & $\begin{array}{l}\text { - Widely availability } \\
\text { - High sensitivity } \\
\text { - High accuracy }\end{array}$ & $\begin{array}{l}\text { - Radiation } \\
\text { exposure } \\
\text { - Low specificity } \\
\text { - No extraluminal } \\
\text { information }\end{array}$ & $\begin{array}{l}\text { - Enema through ileostomy or } \\
\text { fistulography can be rapidly } \\
\text { performed for complications } \\
\text { appraisal }\end{array}$ & $\begin{array}{l}\text { - Barium follow-through provides a visualization } \\
\text { of both small and large bowel loops } \\
\text { - Barium enema offers a wide colonic } \\
\text { representation with additional opacification of } \\
\text { the last ileal loop }\end{array}$ \\
\hline US & $\begin{array}{l}\text { - Radiation free } \\
\text { - Wide availability } \\
\text { - Low healthcare costs }\end{array}$ & $\begin{array}{l}\text { - Dependence } \\
\text { on operator's } \\
\text { expertise } \\
\text { - Prolonged exam } \\
\text { time } \\
\text { - Low field of view }\end{array}$ & $\begin{array}{l}\text { - Color-Doppler provides vascular } \\
\text { information } \\
\text { - CEUS offers additional value in } \\
\text { abscess recognition } \\
\text { - Elastosonography provides } \\
\text { information about fibrotic } \\
\text { involution }\end{array}$ & $\begin{array}{l}\text { - Ileum can be easily evaluated, while colonic } \\
\text { evaluation can be impaired by feces and } \\
\text { meteorism. }\end{array}$ \\
\hline CTE & $\begin{array}{l}\text { - Comprehensive } \\
\text { evaluation } \\
\text { - High sensitivity and } \\
\text { accuracy rates } \\
\text { - Low scan times }\end{array}$ & $\begin{array}{c}\text { - Radiation } \\
\text { exposure }\end{array}$ & $\begin{array}{l}\text { - lodine concentration can } \\
\text { be used as a marker of } \\
\text { inflammation activity on DECT }\end{array}$ & $\begin{array}{l}\text { - Optimal ileum and colonic appraisal } \\
\text { - Jejunal evaluation is typically less clear due to } \\
\text { fast peristalsis }\end{array}$ \\
\hline MRE & $\begin{array}{l}\text { - Lack of radiations } \\
\text { - Comprehensive } \\
\text { evaluation } \\
\text { - High sensitivity and } \\
\text { accuracy rates }\end{array}$ & $\begin{array}{l}\text { - Prolonged scan } \\
\text { times } \\
\text { - General MR } \\
\text { contraindications }\end{array}$ & $\begin{array}{l}\text { - DWI yields inflammation } \\
\text { detection } \\
\text { - Recently developed sequences } \\
\text { (elastography, perfusion and } \\
\text { magnetization transfer) can } \\
\text { provide additional information } \\
\text { about inflammation and } \\
\text { fibrosis differentiation }\end{array}$ & $\begin{array}{l}\text { - Optimal ileum and colonic visualization } \\
\text { - Lack of radiations enables the performance } \\
\text { of repeated scans (obtained at different } \\
\text { timepoints) allowing an optimal bowel } \\
\text { distention on each intestinal district. }\end{array}$ \\
\hline
\end{tabular}

US, ultrasound; CTE, computed tomography enteroclysis; MRE, magnetic resonance enterography; CEUS, contrast-enhanced US; DECT, dual-energy computed tomography; MR, magnetic resonance; DWI, diffusion-weighted imaging.

er diagnostic path. Therefore, the aim of this paper is to afford the clinician a wide overview on radiologic potential in CD assessment, explaining the basic technical notions and the main findings detectable in the use of each. The second purpose is to relate the diagnostic possibilities to the different intestinal tracts, in order to customize the patient management as far as possible on the basis of the clinical picture (Table 1).

\section{IMAGING MODALITIES}

\section{Fluoroscopy}

\section{1) Techniques}

Proximal and distal intestinal loops can be evaluated through different approaches.

Small bowel follow-through (SBFT) is performed through the oral ingestion of a barium solution, whose progression is then recorded obtaining several radiographic images series (Fig. 1). ${ }^{12}$ The duration of the exam strictly depends on intestinal peristalsis and presence of strictures.

SBFT has been reported with a sensitivity of $67 \%$ to $72 \%$. In- terobserver differences in SBFT evaluation have been ascribed to intrinsic drawbacks, such as incomplete visualization of intestinal loops, mainly due to overlap, and suboptimal dilation distal to strictures. ${ }^{13}$

Enteroclysis can be performed through the positioning of a nasoduodenal or nasojejunal catheter and administration of a positive contrast agent which is composed of a water solution of barium and/or methylcellulose. Air can be infused in order to obtain double-contrast images. ${ }^{12,14}$

Despite the invasive procedure of nasoenteric tube positioning, this technique improves dilation of duodenum and jejunal loops and can increase compliance especially in pediatric or elderly patients. Formerly, enteroclysis has demonstrated high degree of sensitivity, specificity and accuracy (respectively up to $100 \%, 98.3 \%$, and $99.3 \%$ ). However, this technique cannot provide any further information on extraintestinal involvement and differential diagnosis, especially at early stages (i.e., from lymphoid hyperplasia and infectious ileitis) and its use has fallen out of favor since the development of cross-sectional imaging. ${ }^{15}$ 

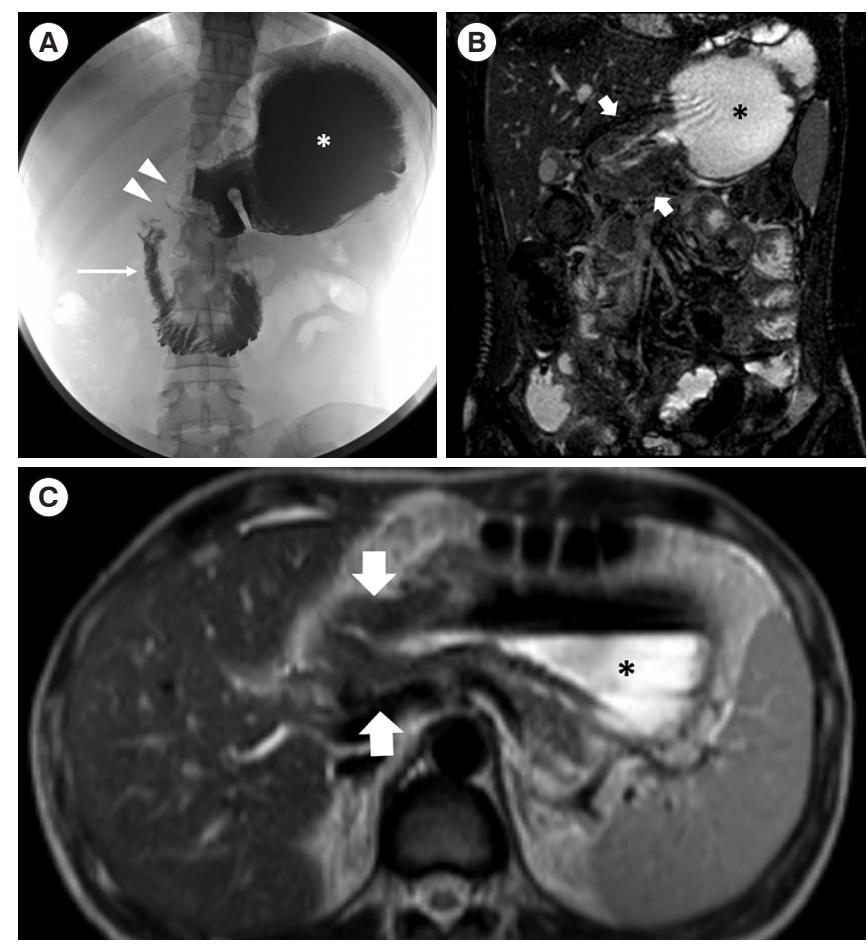

Fig. 1. A 34-year-old female patient affected by Crohn's disease. (A) Barium follow-through photo shows dilation of the gastric lumen (asterisk) with obstruction at the level of the gastric antrum (arrowheads) and distention of duodenal lumen downstream (thin arrow). Coronal B-FFE (B) and axial HASTE T2-weighted (C) confirmed the gastric stenosis caused by a severe thickening of the gastric antrum (thick arrows). B-FFE, balanced-fast field echo; HASTE, half-Fourier acquisition single-shot turbo-spin-echo.

When a distal ileum or colonic involvement is suspected, a trans-rectal approach is preferred and single- or double-contrast enema can be performed. Although the latter allows a more accurate evaluation of the mucosal layer, a single contrast technique is preferred when the presence of a stenosis is probable, since the obstruction can be more easily overcome. ${ }^{16,17}$

Double-contrast barium enema have shown high sensitivity in detecting typical findings of $\mathrm{CD}$, such as discontinuous or eccentric involvement (88\%) and/or discrete ulcers $(67 \%){ }^{18}$

However, differential diagnosis between CD and UC basing on radiological findings remains uncertain in $25 \%$ of the cases. ${ }^{18}$

Despite the widespread availability, the low healthcare costs and the accuracy in evaluating extension of wall lesions, fluoroscopy techniques do not provide any information other than endoluminal caliber, mucosal layer edges and motion rate of the contrast column. ${ }^{14}$

However, they can be useful for other associated clinical conditions. For instance, enema of an ileostomy can be per-
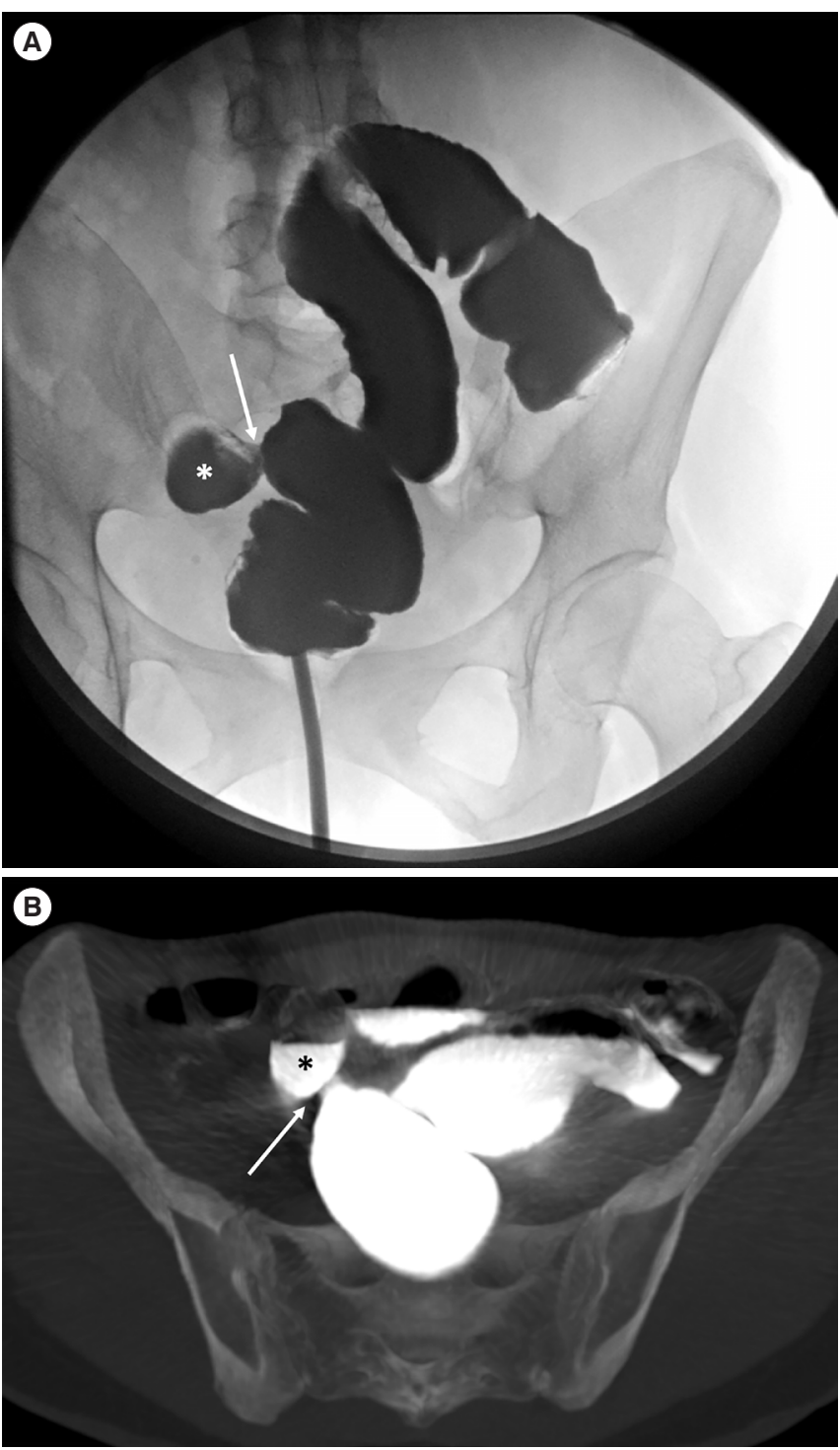

Fig. 2. A 46-year-old female Crohn's disease patient with previous radical colectomy and ileorectal anastomosis. Water-soluble contrast colonic enema $(A)$ showed a fistulous communication (arrow) with an adjacent ileal loop (asterisk), as confirmed in the axial computed tomography-scan maximum intensity projection reconstruction (B).

formed before its removal, in order to detect any loop abnormality, or fistulography for external fistulas path identification, through the administration of an iodinated contrast water solution with a Foley catheter (Fig. 2)..$^{12,16}$

\section{2) CD Findings}

The typical radiological appearance of intestinal mucosa affected by $\mathrm{CD}$ is the "cobblestoning," resulting from an alternation of ulcers, detectable "contrast-plus" images (due to the mu- 
cosal defect and deposition of contrast), and inflammatory hypertrophy of the surrounding tissues, resulting in their prominence and therefore "contrast-minus" images. Other findings include a mucosal "polypoid" appearance, irregular enlargement of intestinal villi, endoluminal stenosis with upstream dilation, linear superficial ulcers which can evolve into fistulas, spaced or attached loops (respectively due to wall thickening/ mesenteric fat hypertrophy or inflammatory adhesions). ${ }^{14-17}$

\section{Ultrasound}

\section{1) Techniques}

Ultrasound (US) is a radiation-free, widespread, low-cost exam whose clinical use in CD patients is usually considered as a first-line diagnostic approach, mainly due to its limited field of view. ${ }^{8}$

A transabdominal approach with a convex probe (3.5-8 MHz) allows to obtain a wide overview of the abdominal cavity (including complications), while the use of linear array probes at higher frequencies (up to $13 \mathrm{MHz}$ ) permits to evaluate the bowel walls in detail, leading to a clear distinction of thickness and stratification. ${ }^{19}$

US has a high level of sensitivity (up to 93\%) in detecting CD bowel lesions, although it is nonspecific. ${ }^{19-21}$ US showed an accuracy similar to computed tomography enteroclysis (CTE) and magnetic resonance enterography (MRE) for CD diagnosis, with a sensitivity and specificity ranging between $75 \%$ and $100 \%{ }^{22-24}$ Moreover, high specificity for strictures, fistulas and abscess has been reported in comparison to surgical specimens. ${ }^{24}$

However, several techniques can significantly increase its diagnostic accuracy. The first one is color-Doppler, which provides important information on vascular flow (Fig. 3). ${ }^{19}$ Contrast-enhanced US (CEUS) is instead performed via the intravenous injection of phospholipid-stabilized microbubbles, whose rapid pulmonary excretion avoids any risk of nephrotoxicity. ${ }^{11}$

CEUS provides a "real time" perfusion appraisal of bowel walls, yielding a distinction of active inflammation from fibrosis and helping in assessing therapy response. ${ }^{8,19}$ A more objective and less "operator-dependent" evaluation can be achieved using dedicated software, which provides quantitative and semiquantitative parameters (such as max intensity peak, time to peak velocity, washout time and intensity) although standardized thresholds are not currently available. ${ }^{11,25}$

An accuracy rate of $97.2 \%$ has been described in differenti-
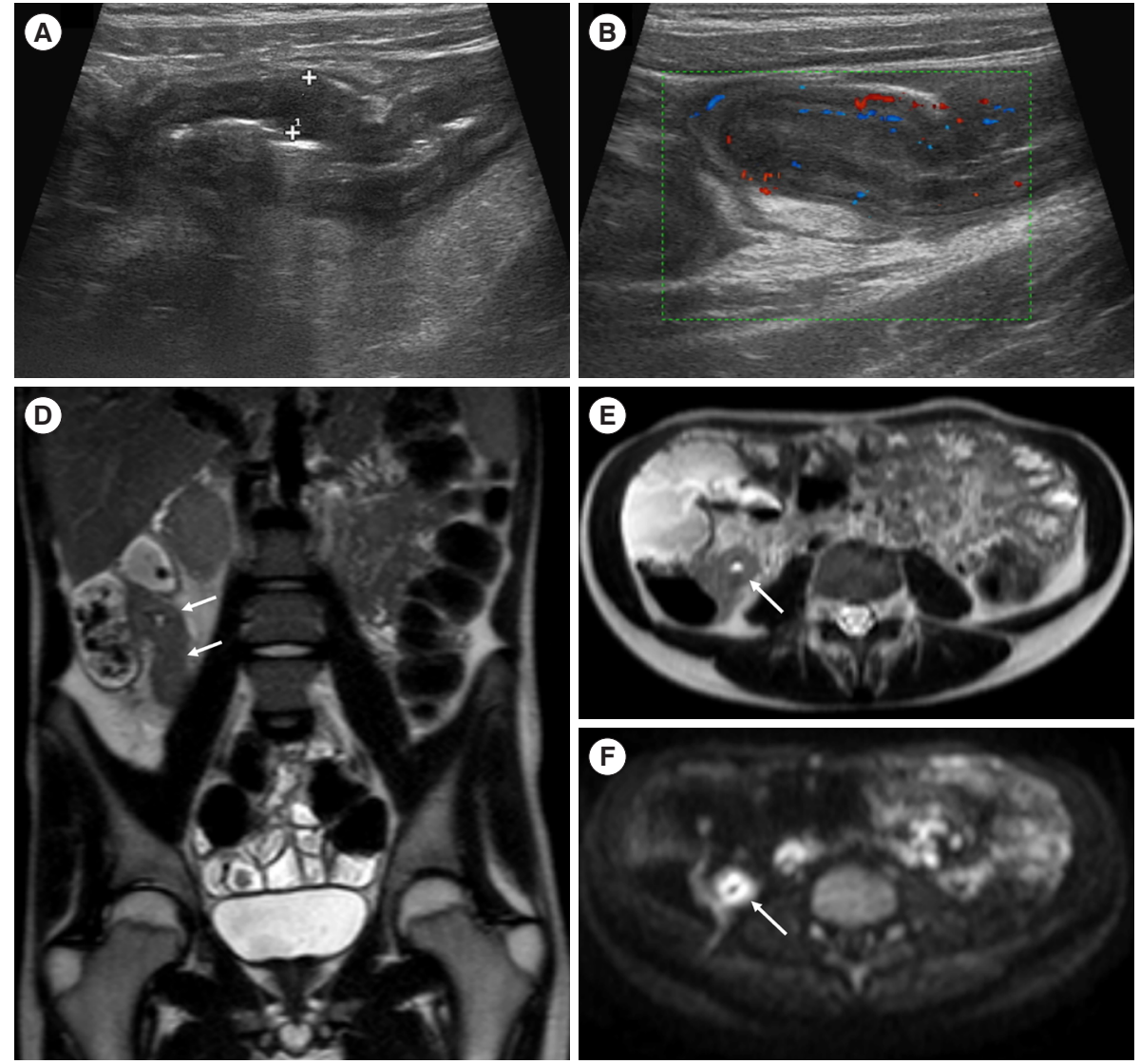
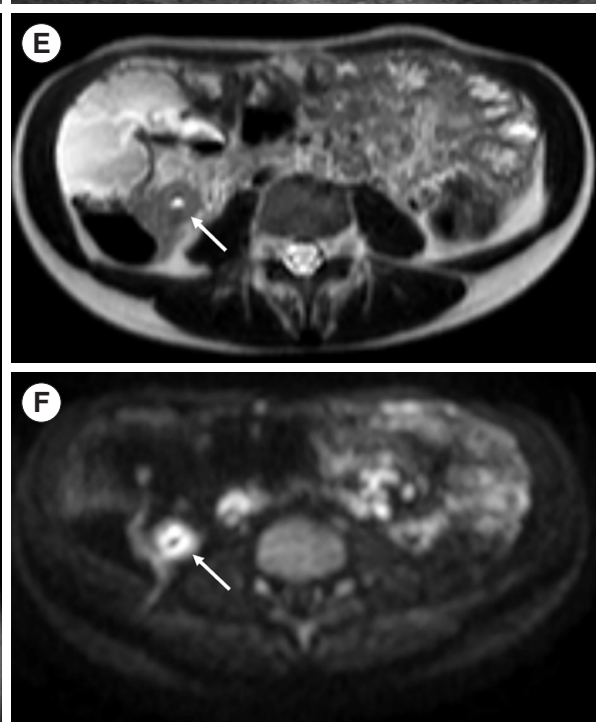

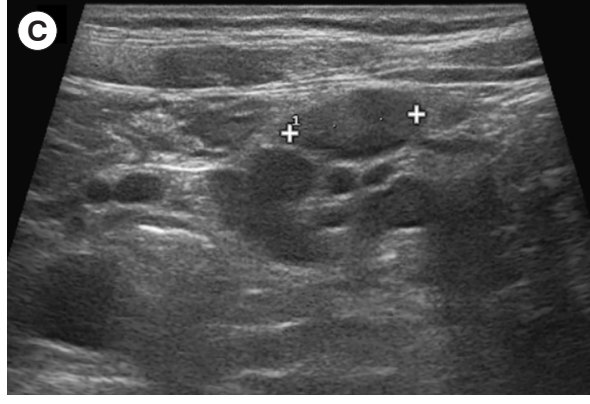

Fig. 3. A 17-year-old male patient was hospitalized for pain in the right iliac fossa. Ultrasound B-mode examination (A) showed a thickened last ileal loop (8 $\mathrm{mm}$ ) with increased vascular signals at colorDoppler (B) and enlarged lymph nodes within the adjacent adipose tissue (C) due to inflammation. The following magnetic resonance enterography confirmed bowel wall thickening (arrows), as visible at coronal HASTE (D), axial HASTE (E) and axial diffusion-weighted imaging (F). HASTE, half-Fourier acquisition single-shot turbospin-echo. 
ating inflammatory phlegmons from intra-abdominal abscesses. $^{25}$

A significant improvement in diagnostic accuracy in sonographic evaluation of $\mathrm{CD}$ has been obtained by small intestine contrast ultrasonography (SICUS). This technique is performed as a conventional transabdominal US after oral contrast administration (125-800 mL of polyethylene glycol water solution). ${ }^{19}$ It allows a better dilation of bowel loops, and an ongoing appraisal of intestinal peristalsis, with increased sensitivity (96\% to $100 \%$ ) in CD lesions as well as complications than conventional US., ${ }^{8,11,26-33}$

Despite undoubted advantages, SICUS is highly time-consuming, considering that the examination time depends on patient peristalsis and operator's experience. ${ }^{11}$

Therefore, SICUS is currently performed in few centers and it is mainly reserved for patients with relative or absolute contraindications to CTE and MRE. ${ }^{11}$

US share-wave and strain elastography are recently developed techniques, sensitive to stiffness of tissues, whose use is now established in evaluation of thyroid nodules. Recent works have demonstrated their feasibility also on thickened bowel walls in CD patients, with the purpose of distinguishing active inflammation from fibrosis. Despite the encouraging results, these techniques have still to be largely and definitely validated. $^{8,26,34-37}$

Undeniable limitations of US techniques remain the limited field of view (especially in identifying complications), the interposition of abdominal fat and/or presence of meteorism. ${ }^{27}$

Nevertheless, US can be useful for evaluation of perianal fistulas and gluteal abscesses, relying on the use of high-frequency probes and on the possibility of 2 different approaches: transperineal and endocavitary (through endoanal and/or endovaginal probes). ${ }^{38}$

\section{2) CD Findings}

US features of CD typically include an increased wall thickness ( $>3 \mathrm{~mm}$ ), with a partial or complete lack of layer stratification ("target sign") and increased vascularization. According to Limberg classification, a color-Doppler semiquantitative assessment of bowel walls vascularity which includes 5 grades, a score $>2$ is considered abnormal. ${ }^{8,39}$ At CEUS, 3 to 4 enhancement patterns, according to the enhanced layers after intravenous contrast agent injection, have been described and several attempts have been made in order to define disease activity. ${ }^{19}$ Concurrent abdominal adenopathies and free fluid can also be detected as well. ${ }^{26}$

\section{Computed Tomography 1) Techniques}

Conventional CT-scan, performed with or without intravenous contrast medium injection, is usually performed in patients with an abrupt onset of a previous unknown CD condition or for acute complications in histologically-confirmed CD patients (i.e., abdominal abscesses, perforation, etc.).

Instead, accurate bowel loops evaluation at CT-scan in a CD patient, whether it is well-known or only clinically suspected, requires the administration of an enteric contrast medium 45-60 minutes before the exam (400-800 $\mathrm{mL}$ of polyethylene glycol for pediatric population; $800-1,500$ for the adults), after a fasting period of $4-6$ hours. ${ }^{40-42}$

The enteric contrast agent can be administrated orally (CTenterography) or via a nasoenteric catheter (CT-enteroclysis).

Intravenous iodine contrast medium is also injected (approximately $1.5 \mathrm{~mL} / \mathrm{kg}$ ). An enteric phase (approximately 6090 seconds after contrast administration) is mandatory, while a multiphasic study is usually reserved for patients with simultaneous conditions (i.e., gastrointestinal bleeding, liver abscesses, etc.). ${ }^{42}$

The main advantage of CT is the possibility of three-dimensional (3D) reconstructions that can be manually managed by the radiologist, and their related tools, including maximum intensity projection, useful for vascular assessment, and volume rendering, which can provide information about bowel wall thickening, abnormal mucosal enhancement, relationships among the intestinal loops and extraintestinal complications (Fig. 4). ${ }^{43,44}$

Despite the higher soft tissue contrast of MR, Spektor et al. ${ }^{45}$ demonstrated that the sensitivity of CT in detecting the most relevant acute findings of CD (i.e., abscess, fistula, bowel wall thickening, free fluid, stricture, and bowel obstruction) is substantially similar to MRE. ${ }^{46}$

In comparison with clinical, histologic, and endoscopic features, high rates of sensitivity and specificity (up to 90\%) have been reported for $\mathrm{CD}$ detection. ${ }^{44}$

Dual-energy CT (DECT) is a particular technique that allows tissue decomposition, whose clinical significance has been already established for abdominal as well as extra-abdominal conditions. ${ }^{47}$

Also, in the CD field, DECT can provide useful information concerning iodine density, which seems to be related to disease activity, and better visual appraisal through iodine maps reconstruction. ${ }^{48,49}$

The main limit of CT-scan techniques lies in the radiation 

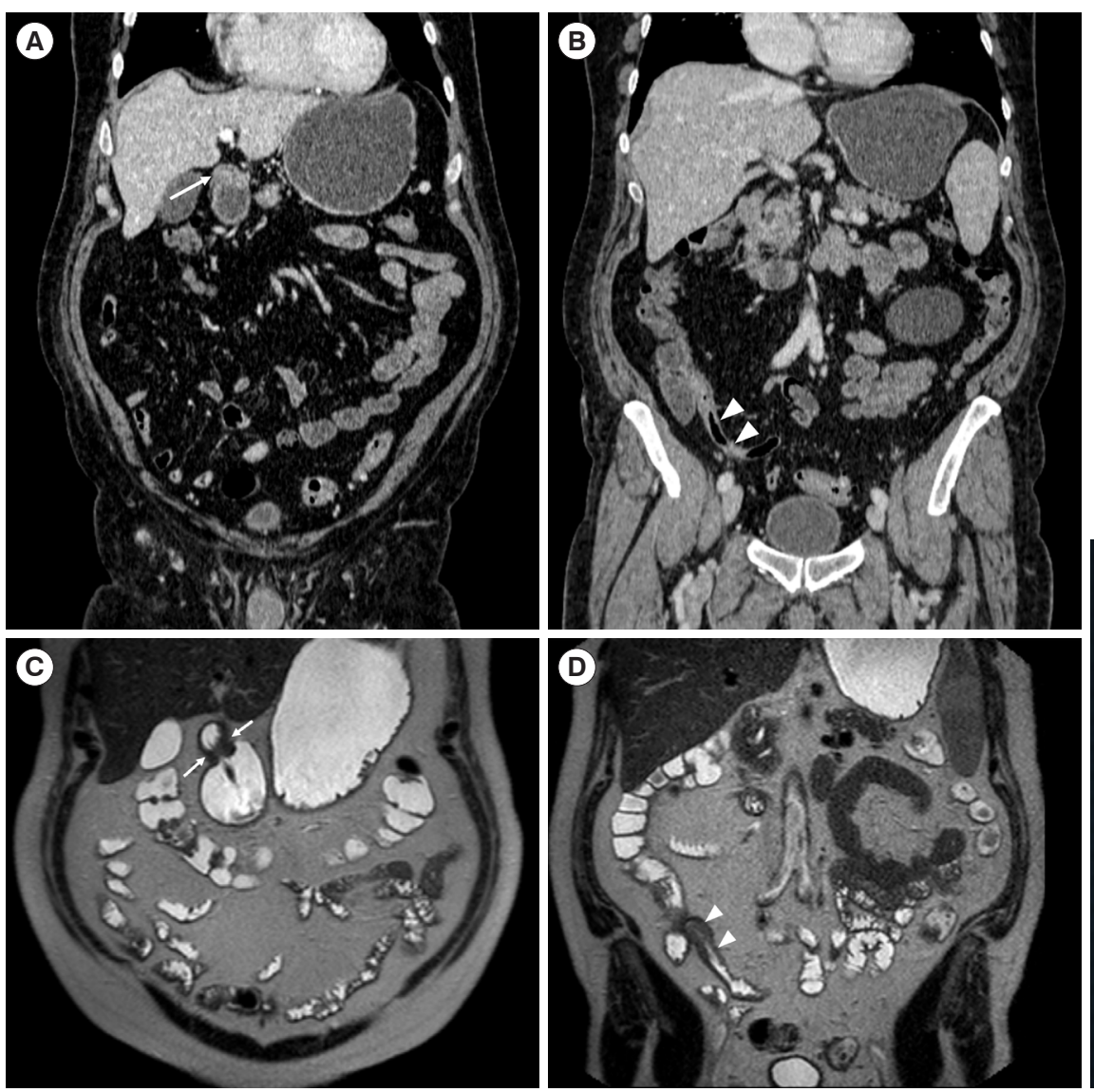

Fig. 4. A 71-year-old male Crohn's disease patient. Coronal computed tomography (CT)enterography scans at different levels $(A, B)$ and magnetic resonance enterography coronal HASTE images $(C, D)$ show wall thickening within the gastric antrum (arrows) and the last ileal loop (arrowheads). Volume-rendering reconstruction (E) is a CT-scan useful tool in providing an overview of the intestinal loops dilation and stenotic points. HASTE, half-Fourier acquisition single-shot turbo-spin-echo.

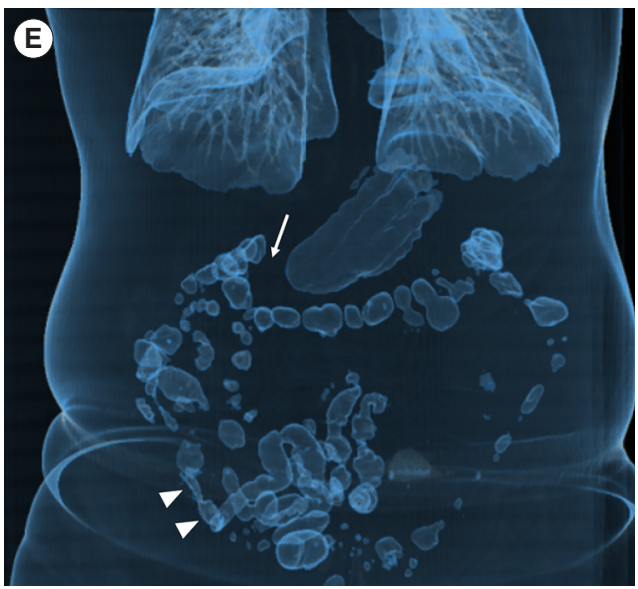

exposure, especially if considering the age of CD patients (the first peak is comprised between the second and the fourth life decades) and the frequent need for follow-up examinations.

Performing "low-dose" protocols is not always a solution, since they are impaired by increased noise artifacts and reduced accuracy. ${ }^{50}$ However, this latter issue has been partially counterbalanced by the recent introduction of iterative reconstructions, alternative filtered back projection tools that allows better-quality reconstructions of images acquired at low voltages. ${ }^{43}$

\section{2) CD Findings}

Bowel wall thickening ( $>3 \mathrm{~mm}$ ) together with mural hyperenhancement are the typical signs of CD. Wall thickening is usually more prominent along the mesenteric side. Layered contrast-enhanced appearance can occur due to submucosal edema, related to active inflammation, or fat infiltration, more indicative of a long-standing disease. This latter scenario can also show presence of strictures with dilation of the proximal loop. On the other hand, lack of stratification can be related to fibrosis or collagen deposition, with lower and more progressive enhancement. Extraintestinal features, such as adenopa- thies, engorgement of vasa recta ("comb sign") and stranding of the surrounding mesenteric fat can be detected. ${ }^{40,41,50-52}$

\section{Magnetic Resonance Imaging 1) Techniques}

Magnetic resonance imaging (MRI) is a radiation-free imaging modality that allows comprehensive evaluation of the bowel loops and the abdominal cavity at the same time. ${ }^{53-55}$ Similar to CT-scan, 2 MR techniques can be distinguished according to mode of oral contrast medium administration: oral ingestion (MR-enterography) or nasojejunal intubation (MR-enteroclysis).$^{56}$ Although the latter permits an improved distention of jejunal loops, it is considered quite uncomfortable and less accepted by the patients. ${ }^{57,58}$ A previous fasting period of 4-6 hours is required; a water solution of oral contrast agent (200-800 $\mathrm{mL}$ for pediatrics; $800-1,000 \mathrm{~mL}$ ) is administrated $30-45$ minutes before the examination.

The typical protocol includes: (1) coronal and axial half-Fourier acquisition single-shot turbo-spin-echo (HASTE; repetition time/echo time [TR/TE]: $\infty / 80 \mathrm{~ms}$ ) without fat-suppression, whose aim is primarily morphologic and addressed to le- 
sion detection and quantification; (2) HASTE (TR/TE: $\infty / 80$ ms) with fat-suppression, helpful in recognizing intra- or extraparietal edema; (3) axial and coronal true-fast imaging with steady-state (True-Fisp; TR/TE: $4.20 / 2.10 \mathrm{~ms}$, flip angle: $60^{\circ}$ ), that decrease intraluminal fluid motion artifacts; (4) axial/coronal diffusion-weighted imaging (DWI) sequences with a diffusion $\mathrm{b}$ factor fixed at 0,400 and $800 \mathrm{~s} / \mathrm{mm}^{2}$, useful for identification of bowel walls inflammations, fistulas, lymph nodes and abdominal abscesses; or (5) ultrafast 3D T1-weighted gradient-echo fat-suppressed, obtained before and after the intravenous injection of gadoterate meglumine (Dotarem) at a dose of $0.2 \mathrm{~mL} / \mathrm{kg}$ body-weight on axial/coronal plane, followed by a bolus of $30 \mathrm{~mL}$ of normal saline, mandatory for enhancement pattern and disease activity evaluation. ${ }^{58,59}$

Additional sequences can also be acquired: (1) coronal thicksection T2-weighted RARE, which magnify the intraluminal fluid signal; (2) cine-MR, helpful in peristalsis assessment; or (3) GE T1-weighted without fat-saturation, for adipose infiltration/lesions.

The patient can be scanned in supine and/or prone position. Supine position is more comfortable for the patient, while prone position would be preferable for the higher pressure on the abdominal cavity, which leads to a better dilation of the loops and decreased motion artifacts.

Similar values of sensitivity, specificity and accuracy have been reported between MRE and colonoscopy, which is currently considered the gold standard in CD diagnosis (respectively $82,80,81$ vs. $85,85,92) .{ }^{60}$

Future perspectives come with the recent development of new techniques, such as magnetization transfer and MR-elastography, whose final purposes lie in a clear differentiation of active inflammation from fibrosis. ${ }^{61}$ MRE has also been successfully applied in intestinal conditions other than $\mathrm{CD}^{62-64}$ However, this concept implies that, similarly to other imaging modalities, the radiological findings are not pathognomonic of $\mathrm{CD}$ and correlation with laboratory tests and endoscopic features is mandatory for a definitive diagnosis.

Evaluation of the perianal fistulas and abscesses requires a specific study of the anal canal, without the need of an enteric contrast agent administration. In this case, the protocol is composed of: a sagittal "anatomical" T2-weighted scan of the pelvis, used as a guide for positioning of the following sequences; HASTE T2-weighted images obtained with and without fat saturation on oblique-coronal and oblique-axial planes (respectively parallel and perpendicular to the longitudinal axis of the anal canal); real-axial or axial-oblique DWI; real-axial or axial- oblique GE T1-weighted scans with fat-saturation obtained before and after intravenous gadolinium $(\mathrm{Gd})$ injection $(0.2$ $\mathrm{mL} / \mathrm{kg})^{65.66}$

\section{2) CD Findings}

Typical features of $\mathrm{CD}$ are wall thickening ( $>3 \mathrm{~mm}$ ) with mural hyperenhancement (layered or homogeneous), intramural edema, strictures with upstream dilation, ulcerations, high signal on DWI images. ${ }^{67}$ Considering that a clear distinction between active inflammation and fibrosis result in significant implications for treatment management of such patients, the evaluation of these findings leads to the establishment of different activity indexes (magnetic resonance index of activity, MaRIA; Crohn's disease activity score, CDAS; MRE global score, MEGS). ${ }^{67-71}$ Briefly, 4 main disease patterns have been identified on MRE: "active inflammation," characterized by hyperintensity of bowel wall on T2-weighted scans (related to edema, restricted diffusivity of water molecules, intense (homogeneous or stratified) enhancement, co-existence of "comb sign" and adenopathies; "fistulizing and perforating," in which, beside the previous findings, sinus tracts, fistulas or abdominal abscesses are found; "fibrostenotic," with iso- or hypointensity of bowel wall thickening on T2-weighted images, no restriction, lower and progressive enhancement; "reparative and regenerative," in which no restriction nor hyperenhancement can be detected. ${ }^{58,72}$ Main limitations of MRE are the prolonged scan time (25-45 minutes), healthcare costs, and sufficient luminal distension, necessary for avoiding artifacts.

\section{INTESTINAL TRACTS}

\section{Proximal Segments}

Although upper gastrointestinal tract localization has been reported in up to $4 \%$ of CD patients, the incidence is undoubtedly higher, considering the number of incidental lesions reported on endoscopy in asymptomatic patients. In most of cases, gastroduodenal lesions are simultaneous with small or large bowel involvement and usually occur in the antrum, pylorus and proximal part of duodenum. ${ }^{73-75}$ Typical features of upper gastrointestinal tract involvement at radiological imaging include short strictures with stenosis, whereas fistulas, though seldom present, seem to origin from other portions of the intestinal tract. Generally related symptoms include epigastric pain, nausea and vomiting, weight loss, intraluminal bleeding However, gastroduodenal localizations are usually asymptomatic and might easily go undetected on cross-sectional imag- 
ing. ${ }^{73,74}$ Main differential diagnosis must include peptic ulcer disease, Menetrier disease, Zollinger-Ellison's syndrome, gastrinoma, tuberculosis, lymphoma, and amyloidosis. ${ }^{76}$ In proximal segments, gastroduodenal endoscopy is paramount for lesion detection. Barium follow-through can be helpful in detection of stenosis with dilation of the upstream segment, however without providing any extra-luminal information. US is significantly impaired by superimposition of transverse colon and intestinal meteorism. CTE and MRE are highly accurate for a comprehensive evaluation of this portion of the intestinal tract. However, a rapid progression of the oral contrast agent or the presence of non-stenotic wall lesions lead to risk of underestimation. The positioning of a nasoenteric tube allows a better dilation, although this procedure is invasive and uncomfortable. In this sense, MR-fluoroscopy sequences, obtained simultaneously to administration of oral contrast agent, can be performed in order to overcome this limitation. ${ }^{77}$ Moreover, MR does not use ionizing radiations and therefore multiple scan series can be safely acquired.

\section{Jejunum}

Jejunal loops mainly lie in the left upper abdominal quadrant and their involvement in CD has a significantly different incidence comparing Eastern (16.7\%) and European Countries $(3.8 \%){ }^{78}$ Jejunal loops are characterized by fast peristalsis with suboptimal distension and possibility of lesion underestimation (due to the luminal collapse) or false-positive findings (i.e., restricted diffusion).$^{51}$ Due to its real-time appraisal, SICUS has demonstrated a higher sensitivity than standard US, from $80 \%$ to $100 \%$ respectively. However, this improvement comes at the cost of a significantly increase of evaluation time (from 25 to 60 minutes). ${ }^{8}$ Improved jejunal dilation on CTE and MRE is usually achieved through the positioning of a nasoduodenal tube. MRE is generally preferable due to its radiation-free evaluation and the possibility of acquiring repeated scans at a different distention degree. MR-fluoroscopy sequence for an ongoing appraisal of the oral contrast medium passage can also be performed. ${ }^{77}$

\section{Ileum}

Ileal loops represent the most frequent CD localization, especially at the level of the terminal portion of the intestinal tract. To date, this phenomenon has been not completely understood, although a combination of genetic and environmental factors, leading to an altered immunologic response and abnormal bacterial colonization of this portion, has been advo- cated ${ }^{79}$ Simultaneous ileal and colonic involvement is reported in up to $50 \%$ of CD patients, while only $30 \%$ shows a solitary small bowel affection. Differential diagnosis includes a number of pathologic conditions (from infections to vasculitides and malignancies) and correlation with laboratory tests and endoscopy is compulsory. ${ }^{80,81}$ However, due to the length and winding course, the ileum also represents the portion of the intestinal tract which is most difficult to explore with endoscopic techniques. Therefore, especially in this tract, radiological imaging plays a pivotal role in providing a wider evaluation and comprehensive disease assessment. Easily available, safe and at low-cost, US (and SICUS in particular) has high sensitivity in lesion detection, especially in the most distal part of the ileum. However, the evaluation of the whole ileal loops may require prolonged examination times and strictly depends on the operator's expertise. Moreover, the presence of shortlength skip lesions may be underrated. CTE and MRE allow a comprehensive and more objective evaluation of this intestinal segment. Their capability in detecting intramural or proximal ileum lesions can exceed endoscopy in up to $50 \%$ of CD patients. $^{51,82}$

\section{Colon}

Over $60 \%$ of CD patients have wall lesions of the colon, $15 \%$ to $20 \%$ of which presenting as an isolated colonic involvement. ${ }^{83}$ An increased rate has been registered over the years for this latter form, and specific genetics and pathologic features, serologic and environmental factors, different from the ileo or ileocolonic CD forms as well as from UC, have been identified. ${ }^{84,85}$ Moreover, a less aggressive behavior has been described for this form, hence the reason why is taking place the definition of "third IBD" for colonic isolated CD forms. ${ }^{84,85}$ Differential diagnosis of CD colonic involvement must include colon malignancies, which in CD patients have an increased risk of occurrence and can be detected as mass-like or circumferential thickening of the colorectal wall. ${ }^{86}$ Barium enema can provide information concerning intraluminal content, including presence of strictures or fistulas. US can undoubtedly evaluate the ileocecal region and sigma, but less easily the right and left colon. Generally, right and left flexures are difficultly to identify and evaluate. ${ }^{19}$ The colonic frame is necessarily included in CTE and MRE exams, and therefore colonic lesions can be easily detected as long as the bowel lumen is well distended. Otherwise, DWI may be impaired by low specificity and can lead to false-positive results, especially when colonic water enema is not performed. ${ }^{87}$ 


\section{Anal Canal}

Involvement of the anal canal is reported in $17 \%$ to $43 \%$ of CD patients. ${ }^{88}$ Perianal fistulas have been classified by Parks and their detection as well as the identification of their path is crucial for subsequent surgical management. Trans-perineal US is a simple, cost-effective, and less invasive diagnostic modality which allows a real time appraisal of perianal fistulae and gluteal abscesses. However, the use of a high-frequency probe, whilst increasing contrast and spatial resolution accuracy, has a limited depth of acoustic waves penetration (approximately $6 \mathrm{~cm}$ ). Therefore, while external opening of a fistula can be easily evaluated, the internal sphincter is difficult to detected. ${ }^{89}$ On the other hand, US performed with and endocavitary approach (using endoanal or transvaginal probes) can give more detailed information on the deepest portion of the fistula. ${ }^{90.92}$ Although both of the 2 evaluation methods can provide useful information, a combination of them would be ideal for an exhaustive assessment. Furthermore, vascular information pro-
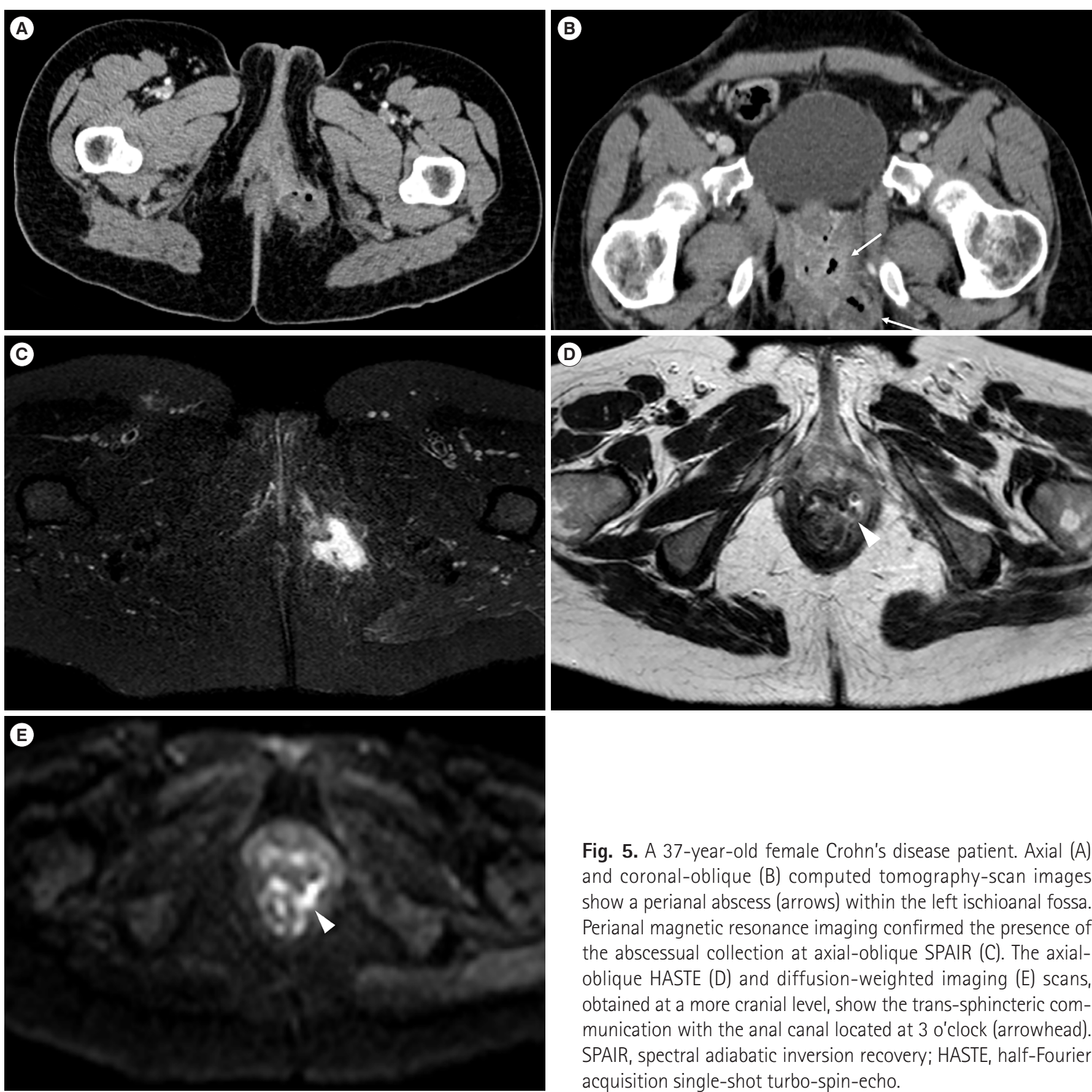

Fig. 5. A 37-year-old female Crohn's disease patient. Axial (A) and coronal-oblique (B) computed tomography-scan images show a perianal abscess (arrows) within the left ischioanal fossa. Perianal magnetic resonance imaging confirmed the presence of the abscessual collection at axial-oblique SPAIR (C). The axialoblique HASTE (D) and diffusion-weighted imaging (E) scans, obtained at a more cranial level, show the trans-sphincteric communication with the anal canal located at 3 o'clock (arrowhead). SPAIR, spectral adiabatic inversion recovery; HASTE, half-Fourier acquisition single-shot turbo-spin-echo. 
vided by color-Doppler can be useful in distinguishing active inflammation from fibrosis. ${ }^{90-92}$

According to recent recommendations, CTE and MRE must include the anal canal within the field of view to analyze any suspicion of a perianal involvement. ${ }^{51}$ However, for the evaluation of the anal canal region, different and specific studies have to be obtained especially in view of future surgical management. CT-scan, although allowing a fast and 3D appraisal, is impaired by low diagnostic accuracy. CT-fistulography, obtained by administration of a prepared solution into the fistula (1 mL Gastrografin in $10 \mathrm{~mL}$ of normal saline) can complement information about the path of the fistula. ${ }^{93,94}$ Perianal MRI can be considered the most comprehensive imaging modality in evaluation of perianal fistulas. Other than the lack of invasiveness and patient preparation, it allows a wide assessment of the anal canal as well as the adjacent structures, detecting the route of fistulas and the presence of abscesses. Moreover, the performance of sequences such as T2-weighted with fat saturation, DWI and contrast-enhanced T1-weighted with fat-saturation, lead to detection of signs of inflammation (i.e., edema, high cellular density, intense enhancement) and therefore distinction of disease activity (Fig. 5).92,95,96

\section{ABDOMINAL CAVITY}

Partially or extensively, the abdominal cavity is necessarily included in all radiological examinations performed for CD assessment, except with fluoroscopic techniques. As easily understandable, US has limited diagnostic potential in evaluating the abdominal cavity. Lymph nodes, mesenteric fat hypertrophy and free fluid are the main findings that are sonographically detectable..$^{19}$ According to their capability in vascularity assessment, perivisceral phlegmons and abscesses can be detected using color-Doppler and CEUS. ${ }^{8,19}$ SICUS may also add important information about internal fistulas and strictures. ${ }^{11}$ However, even if already detected in US, further and wider characterization of intestinal and extraintestinal complications through CT-scan and MRI is often required. Both techniques allow a detailed appraisal of complications, accurate lesion measurements and comprehensive estimation of the peritoneal cavity as well as retroperitoneal spaces involvement. ${ }^{50}$ Nevertheless, concurrent extraintestinal complications involving the parenchymal organs (i.e., liver abscesses, sclerosing cholangitis, mesenteric vascular thromboses, cholelithiasis and nephrolithiasis, pancreatitis, sacroiliitis, etc.) can be detected at the same time. ${ }^{40,41}$

\section{CONCLUSION}

Diagnostic management of CD patients can be challenging considering the huge variety of clinical aspects and the number of imaging modalities and techniques currently available. Endoscopy with biopsy still stand as the reference standard for a definitive diagnosis. The choice of a specific radiological examination depends on patient's symptoms and the imaging modalities available. CTE and MRE provide the most of the information related to intestinal and extraintestinal involvement, with the latter furthermore free from radiation exposure. A good knowledge of radiological imaging modalities and their clinical application is mandatory for an appropriate patient management.

\section{ADDITIONAL INFORMATION}

\section{Funding Source}

The authors received no financial support for the research, authorship, and/or publication of this article.

\section{Conflict of Interest}

No potential conflict of interest relevant to this article was reported.

\section{Author Contribution}

Conceptualization: Cicero G. Supervision: Mazziotti S. Writing - original draft: Cicero G. Writing - review \& editing: Mazziotti S. Approval of final manuscript: all authors.

\section{ORCID}

Cicero G https://orcid.org/0000-0001-9414-4627

Mazziotti S https://orcid.org/0000-0001-9866-569X

\section{REFERENCES}

1. Torres J, Mehandru S, Colombel JF, Peyrin-Biroulet L. Crohn's disease. Lancet 2017;389:1741-1755.

2. GBD 2017 Inflammatory Bowel Disease Collaborators. The global, regional, and national burden of inflammatory bowel disease in 195 countries and territories, 1990-2017: a systematic analysis for the Global Burden of Disease Study 2017. Lancet Gastroenterol Hepatol 2020;5:17-30.

3. Lee SH, Kwon JE, Cho ML. Immunological pathogenesis of inflammatory bowel disease. Intest Res 2018;16:26-42.

4. Kim JM, Cheon JH. Pathogenesis and clinical perspectives of 
extraintestinal manifestations in inflammatory bowel diseases. Intest Res 2020;18:249-264.

5. Levine JS, Burakoff R. Extraintestinal manifestations of inflammatory bowel disease. Gastroenterol Hepatol (N Y) 2011;7: 235-241.

6. Pokala A, Shen B. Update of endoscopic management of Crohn's disease strictures. Intest Res 2020;18:1-10.

7. Hirai F. Current status of endoscopic balloon dilation for Crohn's disease. Intest Res 2017;15:166-173.

8. Kucharzik T, Maaser C. Intestinal ultrasound and management of small bowel Crohn's disease. Therap Adv Gastroenterol 2018;11:1756284818771367.

9. Maaser C, Sturm A, Vavricka SR, et al. ECCO-ESGAR Guideline for Diagnostic Assessment in IBD Part 1: initial diagnosis, monitoring of known IBD, detection of complications. J Crohns Colitis 2019;13:144-164.

10. Sturm A, Maaser C, Calabrese E, et al. ECCO-ESGAR Guideline for Diagnostic Assessment in IBD Part 2: IBD scores and general principles and technical aspects. J Crohns Colitis 2019; 13:273-284.

11. Mocci G, Migaleddu V, Cabras F, et al. SICUS and CEUS imaging in Crohn's disease: an update. J Ultrasound 2017;20:1-9.

12. Fichera A, Krane MK. Crohn's disease: basic principles. Cham: Springer International Publishing, 2015.

13. Lee SS, Kim AY, Yang SK, et al. Crohn disease of the small bowel: comparison of CT enterography, MR enterography, and small-bowel follow-through as diagnostic techniques. Radiology 2009;251:751-761.

14. Gatta G, Di Grezia G, Di Mizio V, et al. Crohn's disease imaging: a review. Gastroenterol Res Pract 2012;2012:816920.

15. Maglinte DD, Chernish SM, Kelvin FM, O'Connor KW, Hage JP. Crohn disease of the small intestine: accuracy and relevance of enteroclysis. Radiology 1992;184:541-545.

16. Levine MS, Ramchandani P, Rubesin S. Practical fluoroscopy of the GI and GU tracts. Cambridge: Cambridge University Press, 2012.

17. Kelvin FM, Oddson TA, Rice RP, Garbutt JT, Bradenham BP. Double contrast barium enema in Crohn's disease and ulcerative colitis. AJR Am J Roentgenol 1978;131:207-213.

18. Margulis AR. Editorial: the overlapping spectrum of radiologic signs in chronic ulcerative colitis and Crohn's disease of the colon. Calif Med 1973;119:49-50.

19. Carnevale Maffè G, Brunetti L, Formagnana P, Corazza GR. Ultrasonographic findings in Crohn's disease. J Ultrasound 2014;18:37-49.

20. Hirche TO, Russler J, Schröder O, et al. The value of routinely performed ultrasonography in patients with Crohn disease. Scand J Gastroenterol 2002;37:1178-1183.

21. Calabrese E, Maaser C, Zorzi F, et al. Bowel ultrasonography in the management of Crohn's disease: a review with recommendations of an international panel of experts. Inflamm Bowel Dis 2016;22:1168-1183.

22. Greenup AJ, Bressler B, Rosenfeld G. Medical imaging in small bowel Crohn's disease-computer tomography enterography, magnetic resonance enterography, and ultrasound: "which one is the best for what?". Inflamm Bowel Dis 2016;22:12461261.

23. Panés J, Bouzas R, Chaparro M, et al. Systematic review: the use of ultrasonography, computed tomography and magnetic resonance imaging for the diagnosis, assessment of activity and abdominal complications of Crohn's disease. Aliment Pharmacol Ther 2011;34:125-145.

24. Rimola J. Cross-sectional imaging in Crohn's disease. Cham: Springer, 2019.

25. Ripollés T, Martínez-Pérez MJ, Paredes JM, Vizuete J, GarcíaMartínez E, Jiménez-Restrepo DH. Contrast-enhanced ultrasound in the differentiation between phlegmon and abscess in Crohn's disease and other abdominal conditions. Eur J Radiol 2013;82:e525-e531.

26. Conti CB, Giunta M, Gridavilla D, Conte D, Fraquelli M. Role of bowel ultrasound in the diagnosis and follow-up of patients with Crohn's disease. Ultrasound Med Biol 2017;43:725-734.

27. Gasche C, Moser G, Turetschek K, Schober E, Moeschl P, Oberhuber G. Transabdominal bowel sonography for the detection of intestinal complications in Crohn's disease. Gut 1999;44: $112-117$.

28. Pallotta N, Tomei E, Viscido A, et al. Small intestine contrast ultrasonography: an alternative to radiology in the assessment of small bowel disease. Inflamm Bowel Dis 2005;11:146-153.

29. Calabrese E, La Seta F, Buccellato A, et al. Crohn's disease: a comparative prospective study of transabdominal ultrasonography, small intestine contrast ultrasonography, and small bowel enema. Inflamm Bowel Dis 2005;11:139-145.

30. Parente F, Greco S, Molteni M, et al. Oral contrast enhanced bowel ultrasonography in the assessment of small intestine Crohn's disease: a prospective comparison with conventional ultrasound, X ray studies, and ileocolonoscopy. Gut 2004;53: 1652-1657.

31. Calabrese E, Zorzi F, Pallone F. Ultrasound in Crohn's disease. Curr Drug Targets 2012;13:1224-1233.

32. Pallotta N, Civitelli F, Di Nardo G, et al. Small intestine contrast ultrasonography in pediatric Crohn's disease. J Pediatr 2013; 
163:778-784.

33. Farmer RG, Whelan G, Fazio VW. Long-term follow-up of patients with Crohn's disease: relationship between the clinical pattern and prognosis. Gastroenterology 1985;88:1818-1825.

34. Baumgart DC, Müller HP, Grittner U, et al. US-based real-time elastography for the detection of fibrotic gut tissue in patients with stricturing Crohn disease. Radiology 2015;275:889-899.

35. Dillman JR, Stidham RW, Higgins PD, et al. Ultrasound shear wave elastography helps discriminate low-grade from highgrade bowel wall fibrosis in ex vivo human intestinal specimens. J Ultrasound Med 2014;33:2115-2123.

36. Fraquelli M, Branchi F, Cribiù FM, et al. The role of ultrasound elasticity imaging in predicting ileal fibrosis in Crohn's disease patients. Inflamm Bowel Dis 2015;21:2605-2612.

37. Rimola J, Capozzi N. Differentiation of fibrotic and inflammatory component of Crohn's disease-associated strictures. Intest Res 2020;18:144-150.

38. Stewart LK, McGee J, Wilson SR. Transperineal and transvaginal sonography of perianal inflammatory disease. AJR Am J Roentgenol 2001;177:627-632.

39. Limberg B, Osswald B. Diagnosis and differential diagnosis of ulcerative colitis and Crohn's disease by hydrocolonic sonography. Am J Gastroenterol 1994;89:1051-1057.

40. Baumgart DC. Crohn's disease and ulcerative colitis. Cham: Springer International Publishing AG, 2017.

41. Huprich JE, Fletcher JG. CT enterography: principles, technique and utility in Crohn's disease. Eur J Radiol 2009;69:393397.

42. Baker ME, Hara AK, Platt JF, Maglinte DD, Fletcher JG. CT enterography for Crohn's disease: optimal technique and imaging issues. Abdom Imaging 2015;40:938-952.

43. Raman SP, Horton KM, Fishman EK. Computed tomography of Crohn's disease: the role of three dimensional technique. World J Radiol 2013;5:193-201.

44. Deepak P, Fletcher JG, Fidler JL, Bruining DH. Computed tomography and magnetic resonance enterography in Crohn's disease: assessment of radiologic criteria and endpoints for clinical practice and trials. Inflamm Bowel Dis 2016;22:22802288.

45. Spektor M, Mathur M, Santacana G, et al. Does MR enterography offer added value after a recent CT in the evaluation of abdominal pain in Crohn's disease patients? Clin Imaging 2019;54:78-83.

46. Chang CW, Wong JM, Tung CC, Shih IL, Wang HY, Wei SC. Intestinal stricture in Crohn's disease. Intest Res 2015;13:19-26.

47. Cicero G, Ascenti G, Albrecht MH, et al. Extra-abdominal du- al-energy CT applications: a comprehensive overview. Radiol Med 2020;125:384-397.

48. Kim YS, Kim SH, Ryu HS, Han JK. Iodine quantification on spectral detector-based dual-energy CT enterography: correlation with Crohn's disease activity index and external validation. Korean J Radiol 2018;19:1077-1088.

49. Dane B, Duenas S, Han J, et al. Crohn's disease activity quantified by iodine density obtained from dual-energy computed tomography enterography. J Comput Assist Tomogr 2020;44: 242-247.

50. Park MJ, Lim JS. Computed tomography enterography for evaluation of inflammatory bowel disease. Clin Endosc 2013; 46:327-366.

51. Bruining DH, Zimmermann EM, Loftus EV Jr, et al. Consensus recommendations for evaluation, interpretation, and utilization of computed tomography and magnetic resonance enterography in patients with small bowel Crohn's disease. Radiology 2018;286:776-799.

52. Elsayes KM, Al-Hawary MM, Jagdish J, Ganesh HS, Platt JF. CT enterography: principles, trends, and interpretation of findings. Radiographics 2010;30:1955-1970.

53. Cicero G, Mondello S, Wichmann JL, et al. Fast magnetic resonance enterography protocol for the evaluation of patients with Crohn's disease: a pilot study. J Clin Imaging Sci 2020;10: 25.

54. Lee JH, Park YE, Seo N, et al. Magnetic resonance enterography predicts the prognosis of Crohn's disease. Intest Res 2018; $16: 445-457$.

55. Kim SH. Computed tomography enterography and magnetic resonance enterography in the diagnosis of Crohn's disease. Intest Res 2015;13:27-38.

56. Ohtsuka K, Takenaka K, Kitazume Y, et al. Magnetic resonance enterography for the evaluation of the deep small intestine in Crohn's disease. Intest Res 2016;14:120-126.

57. Prassopoulos P, Papanikolaou N, Grammatikakis J, Rousomoustakaki M, Maris T, Gourtsoyiannis N. MR enteroclysis imaging of Crohn disease. Radiographics 2001;21 Spec No:S161S172.

58. Moy MP, Sauk J, Gee MS. The role of MR Enterography in assessing Crohn's disease activity and treatment response. Gastroenterol Res Pract 2016;2016:8168695.

59. Mazziotti S, Blandino A. MR enterography. Berlin: Springer, 2014.

60. Taylor SA, Mallett S, Bhatnagar G, et al. Diagnostic accuracy of magnetic resonance enterography and small bowel ultrasound for the extent and activity of newly diagnosed and relapsed 
Crohn's disease (METRIC): a multicentre trial. Lancet Gastroenterol Hepatol 2018;3:548-558.

61. Pita I, Magro F. Advanced imaging techniques for small bowel Crohn's disease: what does the future hold? Therap Adv Gastroenterol 2018;11:1756283X18757185.

62. D’Angelo T, Gallizzi R, Romano C, Cicero G, Mazziotti S. Magnetic resonance enterography findings of intestinal Behçet disease in a child. Case Rep Radiol 2017;2017:8061648.

63. Cicero G, Blandino A, D'Angelo T, et al. Magnetic resonance enterography appraisal of lupus enteritis: a case report. Radiol Case Rep 2018;13:915-919.

64. Cicero G, Ascenti G, Bottari A, Catanzariti F, Blandino A, Mazziotti S. MR enterography: what is next after Crohn's disease? Jpn J Radiol 2019;37:511-517.

65. Erden A. MRI of anal canal: normal anatomy, imaging protocol, and perianal fistulas: part 1. Abdom Radiol (NY) 2018;43: 1334-1352.

66. Thipphavong S, Costa AF, Ali HA, Wang DC, Brar MS, Jhaveri KS. Structured reporting of MRI for perianal fistula. Abdom Radiol (NY) 2019;44:1295-1305.

67. Guglielmo FF, Anupindi SA, Fletcher JG, et al. Small bowel Crohn disease at CT and MR enterography: imaging atlas and glossary of terms. Radiographics 2020;40:354-375.

68. Gücer FI, Senturk S, Özkanli S, Yilmabasar MG, Köroglu GA, Acar M. Evaluation of Crohn's disease activity by MR enterography: derivation and histopathological comparison of an MR-based activity index. Eur J Radiol 2015;84:1829-1834.

69. Ordás I, Rimola J, Alfaro I, et al. Development and validation of a simplified magnetic resonance index of activity for Crohn's disease. Gastroenterology 2019;157:432-439.

70. Steward MJ, Punwani S, Proctor I, et al. Non-perforating small bowel Crohn's disease assessed by MRI enterography: derivation and histopathological validation of an MR-based activity index. Eur J Radiol 2012;81:2080-2088.

71. Makanyanga JC, Pendsé D, Dikaios N, et al. Evaluation of Crohn's disease activity: initial validation of a magnetic resonance enterography global score (MEGS) against faecal calprotectin. Eur Radiol 2014;24:277-287.

72. Kaushal P, Somwaru AS, Charabaty A, Levy AD. MR enterography of inflammatory bowel disease with endoscopic correlation. Radiographics 2017;37:116-131.

73. Heller MT, Haarer KA, Itri JN, Sun X. Duodenum: MDCT of acute conditions. Clin Radiol 2014;69:e48-e55.

74. Lightner AL. Duodenal Crohn's disease. Inflamm Bowel Dis 2018;24:546-551.

75. Song DJ, Whang IS, Choi HW, Jeong CY, Jung SH. Crohn's dis- ease confined to the duodenum: a case report. World J Clin Cases 2016;4:146-150.

76. Pimentel AM, Rocha R, Santana GO. Crohn's disease of esophagus, stomach and duodenum. World J Gastrointest Pharmacol Ther 2019;10:35-49.

77. Cicero G, D'Angelo T, Bottari A, et al. Superior mesenteric artery syndrome in patients with Crohn's disease: a description of 2 cases studied with a novel magnetic resonance enterography (MRE) procedure. Am J Case Rep 2018;19:431-437.

78. Kim YS, Jung SA. Crohn's disease with jejunal involvement as a predictor of long-term clinical outcomes. Gut Liver 2018; 12:3-4.

79. Caprilli R. Why does Crohn's disease usually occur in terminal ileum? J Crohns Colitis 2008;2:352-356.

80. Goulart RA, Barbalho SM, Gasparini RG, de Carvalho AC. Facing terminal ileitis: going beyond Crohn's disease. Gastroenterology Res 2016;9:1-9.

81. Bojic D, Markovic S. Terminal ileitis is not always Crohn's disease. Ann Gastroenterol 2011;24:271-275.

82. Nehra AK, Sheedy SP, Wells ML, et al. Imaging findings of ileal inflammation at computed tomography and magnetic resonance enterography: what do they mean when ileoscopy and biopsy are negative? J Crohns Colitis 2020;14:455-464.

83. Hedrick TL, Friel CM. Colonic Crohn disease. Clin Colon Rectal Surg 2013;26:84-89.

84. Subramanian S, Ekbom A, Rhodes JM. Recent advances in clinical practice: a systematic review of isolated colonic Crohn's disease: the third IBD? Gut 2017;66:362-381.

85. Soucy G, Wang HH, Farraye FA, et al. Clinical and pathological analysis of colonic Crohn's disease, including a subgroup with ulcerative colitis-like features. Mod Pathol 2012;25:295307.

86. Hristova L, Soyer P, Hoeffel C, et al. Colorectal cancer in inflammatory bowel diseases: CT features with pathological correlation. Abdom Imaging 2013;38:421-435.

87. Kim KJ, Lee Y, Park SH, et al. Diffusion-weighted MR enterography for evaluating Crohn's disease: how does it add diagnostically to conventional MR enterography? Inflamm Bowel Dis 2015;21:101-109.

88. Kelley KA, Kaur T, Tsikitis VL. Perianal Crohn's disease: challenges and solutions. Clin Exp Gastroenterol 2017;10:39-46.

89. Wedemeyer J, Kirchhoff T, Sellge G, et al. Transcutaneous perianal sonography: a sensitive method for the detection of perianal inflammatory lesions in Crohn's disease. World J Gastroenterol 2004;10:2859-2863.

90. Sun Y, Cui LG, Liu JB, Wang JR, Ping H, Chen ZW. Utility of 
$360^{\circ}$ real-time endoanal sonography for evaluation of perianal fistulas. J Ultrasound Med 2018;37:93-98.

91. Arora U, Kedia S, Garg P, et al. Colonic Crohn's disease is associated with less aggressive disease course than ileal or ileocolonic disease. Dig Dis Sci 2018;63:1592-1599.

92. Hwang JY, Yoon HK, Kim WK, et al. Transperineal ultrasonography for evaluation of the perianal fistula and abscess in pediatric Crohn disease: preliminary study. Ultrasonography 2014;33:184-190.

93. Liang C, Lu Y, Zhao B, Du Y, Wang C, Jiang W. Imaging of anal fistulas: comparison of computed tomographic fistulography and magnetic resonance imaging. Korean J Radiol 2014;15:
712-723

94. Liang C, Jiang W, Zhao B, Zhang Y, Du Y, Lu Y. CT imaging with fistulography for perianal fistula: does it really help the surgeon? Clin Imaging 2013;37:1069-1076.

95. O’Malley RB, Al-Hawary MM, Kaza RK, Wasnik AP, Liu PS, Hussain HK. Rectal imaging: part 2, perianal fistula evaluation on pelvic MRI--what the radiologist needs to know. AJR Am J Roentgenol 2012;199:W43-W53.

96. Balcı S, Onur MR, Karaosmanoğlu AD, et al. MRI evaluation of anal and perianal diseases. Diagn Interv Radiol 2019;25:2127. 